\title{
Achados patológicos e imuno-histoquímicos de neoplasmas pulmonares primários em caninos na região metropolitana de Porto Alegre, Rio Grande do Sul ${ }^{1}$
}

\author{
Rafaela A. Caprioli², Fernando F. Argenta ${ }^{2}$, Márcia Elisa Hammerschmitt², \\ Paula R. Pereira ${ }^{2}$, Cíntia de Lorenzo ${ }^{2}$, Saulo P. Pavarini² ${ }^{2}$ David Driemeier ${ }^{2}$ \\ e Luciana Sonne ${ }^{2 *}$
}

\begin{abstract}
Caprioli R.A., Argenta F.F., Hammerschmitt M.E., Pereira P.R., Lorenzo C., Pavarini S.P., Driemeier D. \& Sonne L. 2018. [Pathological and immunohistochemical findings of primary pulmonary neoplams in dogs in the metropolitan region of Porto Alegre, Rio Grande do Sul.] Achados patológicos e imuno-histoquímicos de neoplasmas pulmonares primários em caninos na região metropolitana de Porto Alegre, Rio Grande do Sul. Pesquisa Veterinária Brasileira 38(6):1151-1158. Setor de Patologia Veterinária, Faculdade de Veterinária, Universidade Federal do Rio Grande do Sul, Av. Bento Gonçalves 9090, prédio 42505, Porto Alegre, RS 91540-000, Brazil. E-mail: lusonne@yahoo.com.br

Primary pulmonary neoplasms are uncommon in veterinary medicine; but most frequently affected are dogs. It appears that the number of primary pulmonary neoplasms is increasing in companion animals, due to the increase in life expectancy, improvement of diagnostic techniques and greater contact with air pollutants. This study was realized to identify, analyze the frequency, and to characterize pathological and immunohistochemical findings of primary pulmonary neoplasms in dogs of the metropolitan region of Porto Alegre, Rio Grande do Sul. From January 2003 to June 2016, 6,037 necropsies were performed and in 36 of them primary pulmonary neoplasm was found. The most frequent neoplasm in this study was papillary adenocarcinoma (30.5\%) followed by lepidic adenocarcinoma $(22.2 \%)$. The age of affected dogs ranged from 4 to 16 years, with a mean of 11 years. The frequency of male dogs $(63.9 \%)$ prevailed over females (36.1\%). Through immunohistochemical evaluation all epithelial neoplasms were positive for pancytokeratin, $70.6 \%$ were positive for thyroid-1 transcription factor and $23.5 \%$ had co-expression of pancytokeratin and vimentin. Two mesenchymal tumors showed immunostaining for vimentin and S-100, were negative for smooth-muscle actin and melan-A immunohistochemistry and Masson's trichrome histochemical technique and were diagnosed as malignant peripheral nerve sheath tumors, rarely found as primary lung tumor in animals. If one has difficulty to establish a diagnosis only through routine histological examination, it is necessary to emphasize the importance of the immunohistochemical technique for the diagnosis of primary pulmonary neoplasms in dogs.
\end{abstract}

INDEX TERMS: Immunohistochemistry, pulmonary neoplasm, dogs, Rio Grande do Sul, Brazil, diseases of dogs, lung tumors, lung, pathology.

RESUMO.- Neoplasmas pulmonares primários são pouco frequentes na medicina veterinária, mas a principal espécie afetada é a canina. Acredita-se que o número de neoplasmas

\footnotetext{
${ }^{1}$ Recebido em 20 de abril de 2017.

Aceito para publicação em 12 de maio de 2017.

${ }^{2}$ Setor de Patologia Veterinária (SPV), Faculdade de Veterinária, Universidade Federal do Rio Grande do Sul (UFRGS), Av. Bento Gonçalves 9090, Prédio 42505, Porto Alegre, RS 91540-000, Brasil. *Autor para correspondência: lusonne@yahoo.com.br
}

pulmonares primários vem aumentando em animais de companhia, em decorrência do aumento da expectativa de vida, da melhora nas técnicas de diagnóstico e pelo maior contato com poluentes atmosféricos. Este estudo foi realizado para identificar, analisar a frequência, e caracterizar achados patológicos e imuno-histoquímicos dos neoplasmas pulmonares primários em cães na região metropolitana de Porto Alegre, Rio Grande do Sul. No período de janeiro de 2003 a junho de 2016 foram submetidos à necropsia 6.307 caninos. Destes, 
36 cães tinham diagnóstico de neoplasma pulmonar primário. 0 neoplasma mais frequente neste estudo foi o adenocarcinoma papilar $(30,5 \%)$, seguido do carcinoma lepídico $(22,2 \%)$. A idade dos cães variou de quatro a 16 anos, com média de 11 anos. Observou-se que a frequência de cães machos $(63,9 \%)$ prevaleceu sobre as fêmeas $(36,1 \%)$. Na avaliação imuno-histoquímica, todos os neoplasmas epiteliais foram positivos para pancitoqueratina, $70,6 \%$ foram positivos para fator de transcrição de tireoide-1 e 23,5\% tiveram coexpressão de pancitoqueratina e vimentina. Dois tumores mesenquimais tiveram imunomarcação para vimentina e S-100, foram negativos para a imuno-histoquímica de actina de músculo liso e melan-A e para a técnica histoquímica de tricrômico de Masson e foram diagnosticados como tumor da bainha de nervo periférico maligno, raramente encontrado como tumor primário de pulmão em animais. Quando é difícil estabelecer um diagnóstico apenas com o exame histológico de rotina, é necessário ressaltar a importância da técnica de imuno-histoquímica para o diagnóstico de neoplasmas pulmonares primários em cães.

TERMOS DE INDEXAÇÃO: Imuno-histoquímica, neoplasma pulmonar primário, caninos, Porto Alegre, Rio Grande do Sul, doenças de cães, pulmão, patologia.

\section{INTRODUÇÃO}

Neoplasmas pulmonares primários (NPP) em cães possuem frequência de cerca de 0,1\% (Nielsen 1968) a 0,9\% (Stunzi et al. 1974), portanto são tumores incomuns. Os pulmões frequentemente são alvos de tumores metastáticos devido à alta vascularização e extensa rede de drenagem linfática (Wilson 2017). Tumores como osteossarcoma, hemangiossarcoma, melanoma entre outros, frequentemente, fazem metástases para os pulmões, porém os carcinomas mamários, pela sua alta prevalência em cães, são os tumores que mais comumente causam metástases nestes órgãos (Priebe et al. 2011). Tumores metastáticos podem ser semelhantes histologicamente a tumores pulmonares primários, o que dificulta a determinação da origem das células neoplásicas apenas pelo exame histopatológico de rotina (Dungworth et al. 1999), e, nestes casos, a imuno-histoquímica (IHQ) se torna um exame útil e necessário no diagnóstico de NPP (Ramos-Vara et al. 2005).

Neoplasmas pulmonares primários podem ser divididos em tumores epiteliais ou mesenquimais e são classificados conforme a origem (broncogênico, de glândula bronquial ou bronquioloalveolar), o padrão histológico (adenoide, escamoso, de grandes ou pequenas células) ou uma combinação entre eles (Wilson 2017). A grande maioria é epitelial e maligno. Adenocarcinoma e o carcinoma lepídico são os NPP mais comuns em caninos, diferente dos humanos, nos quais carcinoma anaplásico e carcinoma de células escamosas predominam (Ogilvie et al. 1989, Hahn et al. 1996, Wilson 2017, Ramos-Vara et al. 2005, Polton et al. 2008).

0 maior contato entre o homem e o cão pode, possivelmente, ter aumentado os casos de NPP, devido a altas exposições a fatores carcinogênicos, como fumaça de cigarros, agentes radioativos e poluição (Reif et al. 1992). Esse trabalho tem por objetivo identificar e analisar a frequência dos diferentes casos de NPP em cães, assim como comparar os aspectos patológicos e imuno-histoquímicos destes casos, através do estudo retrospectivo de necropsias de caninos da região metropolitana de Porto Alegre, no Estado do Rio Grande do Sul, Brasil.

\section{MATERIAL E MÉTODOS}

Realizou-se um estudo retrospectivo, através dos registros de necropsias do Setor de Patologia Veterinária da Universidade Federal do Rio Grande do Sul (SPV/UFRGS) no período de janeiro de 2003 a junho de 2016 e foram selecionados caninos com neoplasmas pulmonares. Os cães submetidos à necropsia eram procedentes de hospitais e clínicas veterinárias da região metropolitana de Porto Alegre, Rio Grande do Sul, Brasil. Os protocolos de necropsia foram revisados e os dados, como raça, sexo, idade, achados macroscópicos e histopatológicos foram obtidos através das fichas clínicas.

A inclusão dos casos no estudo foi o diagnóstico definitivo de neoplasma pulmonar primário de acordo com critérios histológicos estabelecidos pelo Wilson (2017). Cães com neoplasmas pulmonares possivelmente metastáticos foram excluídos do estudo.

Amostras teciduais, coletadas durante a necropsia, foram fixadas em solução de formalina $10 \%$, desidratadas em concentrações crescentes de álcool etílico, diafinizadas em xilol, incluídas em parafina, seccionadas em $3 \mu \mathrm{m}$ e coradas pela técnica de hematoxilina e eosina (HE). Nas técnicas de IHQ realizadas foram utilizados os anticorpos anti-pancitoqueratina e anti-fator de transcrição de tireoide-1 (TTF-1) em tumores epiteliais, anti-vimentina nos tumores epiteliais e mesenquimas; e anti-actina de músculo liso, anti-S-100 e anti-Melan A nos tumores mesenquimais. Para cada técnica foram incluídos controles positivos previamente testados. Para controle negativo substituiu-se o anticorpo primário por tampão fosfato-salino (PBS). Dados sobre os anticorpos empregados, recuperação antigênica, método de detecção, cromógeno e controle positivo podem ser visualizados no Quadro 1. Utilizou-se também a técnica histoquímica de tricrômico de Masson em neoplasmas mesenquimais.

\section{RESULTADOS}

\section{Achados epidemiológicos}

No período de janeiro de 2003 a junho de 2016 foram necropsiados 6.307 cães no SPV-UFRGS. Desses, 36 (0,5\%) foram diagnosticados com neoplasma pulmonar primário, não sendo necessariamente esta a causa da morte ou motivo para eutanásia em todos os casos.

A faixa etária dos cães variou de quatro a 16 anos, com média de 11 anos de idade. Em sete casos (19,4\%) a idade não foi informada. Os cães sem raça definida (SRD) foram os mais afetados (19/36; 52,8\%), seguidos pelas raças Teckel (4/36; $11,1 \%)$ e Boxer (4/36; 11,1\%). Dos 36 casos, $23(63,9 \%)$ eram machos e $13(36,1 \%)$ eram fêmeas.

Adenocarcinoma papilar foi o NPP mais encontrado neste estudo, seguido do carcinoma lepídico, carcinoma de grandes células, adenocarcinoma misto, adenocarcinoma acinar, tumor de bainha de nervo periférico maligno, adenocarcinoma sólido, carcinoma adenoescamoso, carcinoma combinado e carcinoma de células escamosas (Quadro 2). Dos adenocarcinomas mistos, três eram uma combinação de adenocarcinoma papilar e acinar e, um deles tinha, além do papilar e acinar, um padrão sólido. No carcinoma combinado foi observado o padrão de adenocarcinoma acinar com carcinoma de pequenas células. 
Quadro 1. Protocolos imuno-histoquímicos realizados para auxiliar o diagnóstico de neoplasmas pulmonares primários

\begin{tabular}{|c|c|c|c|c|c|c|}
\hline Anticorpo & Clone/Código & Recuperação antigênica & Diluição & $\begin{array}{c}\text { Método de } \\
\text { detecção }\end{array}$ & Cromógeno & Controle positivo \\
\hline $\begin{array}{l}\text { Monoclonal Anti- } \\
\text { Pancitoqueratina humana }\end{array}$ & AE1/AE3, Dako & $3 \mathrm{~min} / 125^{\circ} \mathrm{C}$, tampão citrato $\mathrm{pH} 6,0$ & $1: 80$ & MACH4 & DAB & Pele de canino \\
\hline Monoclonal Anti-TTF-1 & 8G7G3/1, Dako & $\begin{array}{l}3 \times 5 \text { min micro-ondas, tampão Tris-EDTA } \\
\text { pH } 8,0\end{array}$ & $1: 400$ & $\mathrm{MACH} 4$ & $\mathrm{DAB}$ & Tireoide de canino \\
\hline Policlonal Anti-S100 & Z0311, Dako & $20 \mathrm{~min} / 100^{\circ} \mathrm{C}$, tampão citrato $\mathrm{pH} 6,0$ & $1: 200$ & $\mathrm{MACH} 4$ & $\mathrm{DAB}$ & $\begin{array}{l}\text { Tumor maligno de } \\
\text { bainha de nervo } \\
\text { periférico }\end{array}$ \\
\hline $\begin{array}{l}\text { Monoclonal Anti-Actina de } \\
\text { músculo liso humano }\end{array}$ & M0851, Dako & $\begin{array}{l}20 \mathrm{~min} / 120^{\circ} \mathrm{C} \text {, tampão tris-EDTA } \\
\text { pH 9,0 }\end{array}$ & $1: 100$ & $\mathrm{MACH} 4$ & $\mathrm{DAB}$ & Intestino canino \\
\hline Monoclonal Anti-Melan-A & A103, Dako & $\begin{array}{l}20 \mathrm{~min} / 100^{\circ} \mathrm{C} \text {, Tris-EDTA } \\
\mathrm{pH} 9,0\end{array}$ & $1: 500$ & $\mathrm{MACH} 4$ & DAB & Melanoma \\
\hline
\end{tabular}

TTF-1 = thyroid transcription factor-1, LSAB-HRP = streptavidin biotin horseradish peroxidase, MACH 4 = Universal HRP Polymer (Biocare), $\mathrm{DAB}=3,3^{\prime}$ Diaminobenzidina (Dako).

Quadro 2. Distribuição dos neoplasmas pulmonares primários de acordo com os diagnósticos histopatológicos

\begin{tabular}{lcc}
\hline \multicolumn{1}{c}{ Classificação histopatológica } & Total & $\%$ \\
\hline Adenocarcinoma papilar & 11 & $30,5 \%$ \\
Carcinoma lepídico & 8 & $22,2 \%$ \\
Carcinoma de grandes células & 5 & $13,9 \%$ \\
Adenocarcinoma misto & 4 & $11,1 \%$ \\
Adenocarcinoma acinar & 2 & $5,5 \%$ \\
Tumor da bainha de nervo periférico maligno & 2 & $5,5 \%$ \\
Adenocarcinoma sólido & 1 & $2,8 \%$ \\
Carcinoma adenoescamoso & 1 & $2,8 \%$ \\
Carcinoma combinado & 1 & $2,8 \%$ \\
Carcinoma de células escamosas & 1 & $2,8 \%$ \\
TOTAL & 36 & $100 \%$
\end{tabular}

\section{Achados macroscópicos}

Macroscopicamente os NPP se apresentaram de três formas distintas: multifocal, por vezes coalescente - com neoplasmas brancacentos a amarelados distribuídos em todos os lobos pulmonares (15/36; 45,4\%) (Fig.1A, 1B); focal - com nódulo ou massa única, brancacenta a amarelada (12/36; 33,3\%) (Fig.1C); e difuso, com pulmões aumentados, não colapsados, acinzentados a amarelados, firmes, afetando todos os lobos (9/36; 25\%) (Fig.1D). Quanto à localização, os tumores focais tiveram maior ocorrência no lobo caudal direito $(6 / 12 ; 50 \%)$, seguido do lobo cranial direito $(3 / 12 ; 25 \%)$ e lobo cranial esquerdo $(2 / 12 ; 16,7 \%)$ e, menos comumente, o lobo médio $(1 / 12 ; 8,3 \%)$.

Em 11 tumores houve metástase. Destes tumores, os mais prevalentes foram o adenocarcinoma $(6 / 11 ; 54,5 \%)$ e o carcinoma de grandes células $(3 / 11 ; 27,3 \%)$, e, com menor frequência o carcinoma lepídico $(1 / 11 ; 9 \%)$ e o carcinoma combinado $(1 / 11 ; 9 \%)$. A ocorrência de metástase predominou nos linfonodos mediastínicos $(5 / 36 ; 13,9 \%)$, nas adrenais $(4 / 36 ; 11,1 \%)$ e nos linfonodos traqueobrônquicos $(3 / 36$; $8,3 \%)$, e, menos comumente, no fígado $(2 / 36 ; 5,5 \%)$, baço $(1 / 36 ; 2,8 \%)$, rim $(1 / 36 ; 2,8 \%)$, encéfalo $(1 / 36 ; 2,8 \%)$, hipófise $(1 / 36 ; 2,8 \%)$, tireoide $(1 / 36 ; 2,8 \%)$ e esôfago $(1 / 36 ; 2,8 \%)$.

\section{Achados histopatológicos}

Os padrões histológicos encontrados em casos de adenocarcinoma $(18 / 36 ; 50 \%)$ variaram de papilar (11/18; 61,1\%) (Fig.2A), acinar (2/18; 11,1\%), sólido $(1 / 18 ; 5,5 \%)$ e misto $(4 / 18 ; 22,2 \%)$. Estes neoplasmas se caracterizaram por invadir e destruir o parênquima pulmonar, substituindo-o pelo padrão histológico predominante (papilar, acinar, sólido ou misto), sustentados por discreto $(10 / 18 ; 55,5 \%)$ a moderado $(8 / 18 ; 44,4 \%)$ estroma fibroso. As células variavam de redondas a colunares, com citoplasma eosinofílico, bem delimitado, núcleo arredondado a ovalado e nucléolo evidente, por vezes, indistinto. 0 pleomorfismo variou de discreto $(9 / 18 ; 50 \%)$ a moderado $(9 / 18 ; 50 \%)$, com baixo índice mitótico (média inferior a uma figura de mitose por campo de 400x). Em meio ao tumor havia fendas de colesterol $(9 / 18 ; 50 \%)$, mucina $(5 / 18 ; 27,8 \%)$, necrose $(11 / 18 ; 61,1 \%)$, invasão de vasos linfáticos $(7 / 18 ; 38,9 \%)$, estruturas basofílicas lamelares $(8 / 18 ; 44,4 \%)$, hemorragia $(5 / 18 ; 27,8 \%)$ e trombose $(4 / 18 ; 22,2 \%)$.

Os carcinomas lepídicos $(8 / 36 ; 22,2 \%)$ se caracterizaram por células neoplásicas em espaços alveolares. Eram sustentadas por estroma de tecido conjuntivo que variou de discreto (5/8; $62,5 \%)$, moderado $(2 / 8 ; 25 \%)$ a acentuado $(1 / 8 ; 12,5 \%)$. As células eram poligonais a arredondadas, com citoplasma eosinofílico, bem delimitado, núcleo redondo a ovalado, e nucléolo, em sua maioria, indistinto, havia pleomorfismo discreto $(2 / 8 ; 25 \%)$ a moderado $(6 / 8 ; 75 \%)$ e mitoses em baixo índice ou ausentes. Foram observadas fendas de colesterol $(2 / 8$; $25 \%)$, necrose $(3 / 8 ; 37,5 \%)$, estruturas basofílicas lamelares $(3 / 8 ; 37,5 \%)$, invasão de vasos $(5 / 8 ; 62,5 \%)$ e trombose $(2 / 8$; $25 \%$ ) intratumorais. Em um caso foram observadas células gigantes multinucleadas neoplásicas $(1 / 8 ; 12,5 \%)$ (Fig.2B).

Os carcinomas de grandes células $(5 / 36 ; 13,9 \%)$ alteravam a arquitetura pulmonar e se caracterizaram por células neoplásicas grandes, redondas a poligonais, com citoplasma amplo e vacuolizado, e limites citoplasmáticos distintos. 0 núcleo era arredondado a ovalado e nucléolo evidente, em sua maioria. Havia acentuado pleomorfismo (5/5; 100\%), inúmeras células gigantes multinucleadas e o índice mitótico variava de uma a duas figuras de mitose por campo de maior aumento (400x). Em meio ao tumor eram 

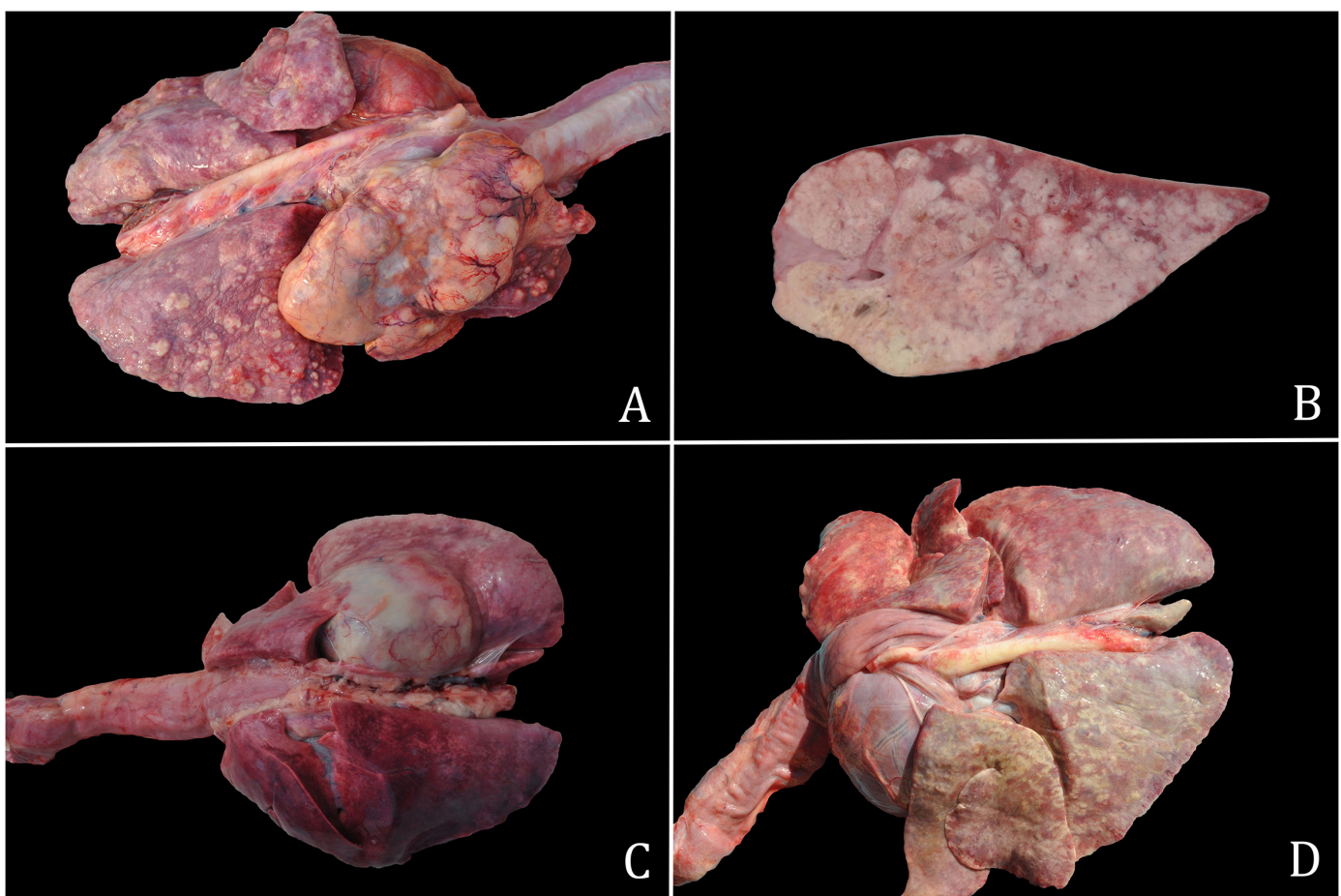

Fig.1. Aspectos macroscópicos dos principais neoplasmas pulmonares primários de cães diagnosticados no SPV/UFRGS. (A) Adenocarcinoma papilar com padrão multifocal, por vezes coalescente, distribuído em todos os lobos pulmonares. (B) Superfície de corte de adenocarcinoma papilar com padrão multifocal a coalescente. (C) Carcinoma lepídico com padrão focal em lobo caudal direito. (D) Carcinoma de grandes células com padrão difuso. Pulmões não colapsados.

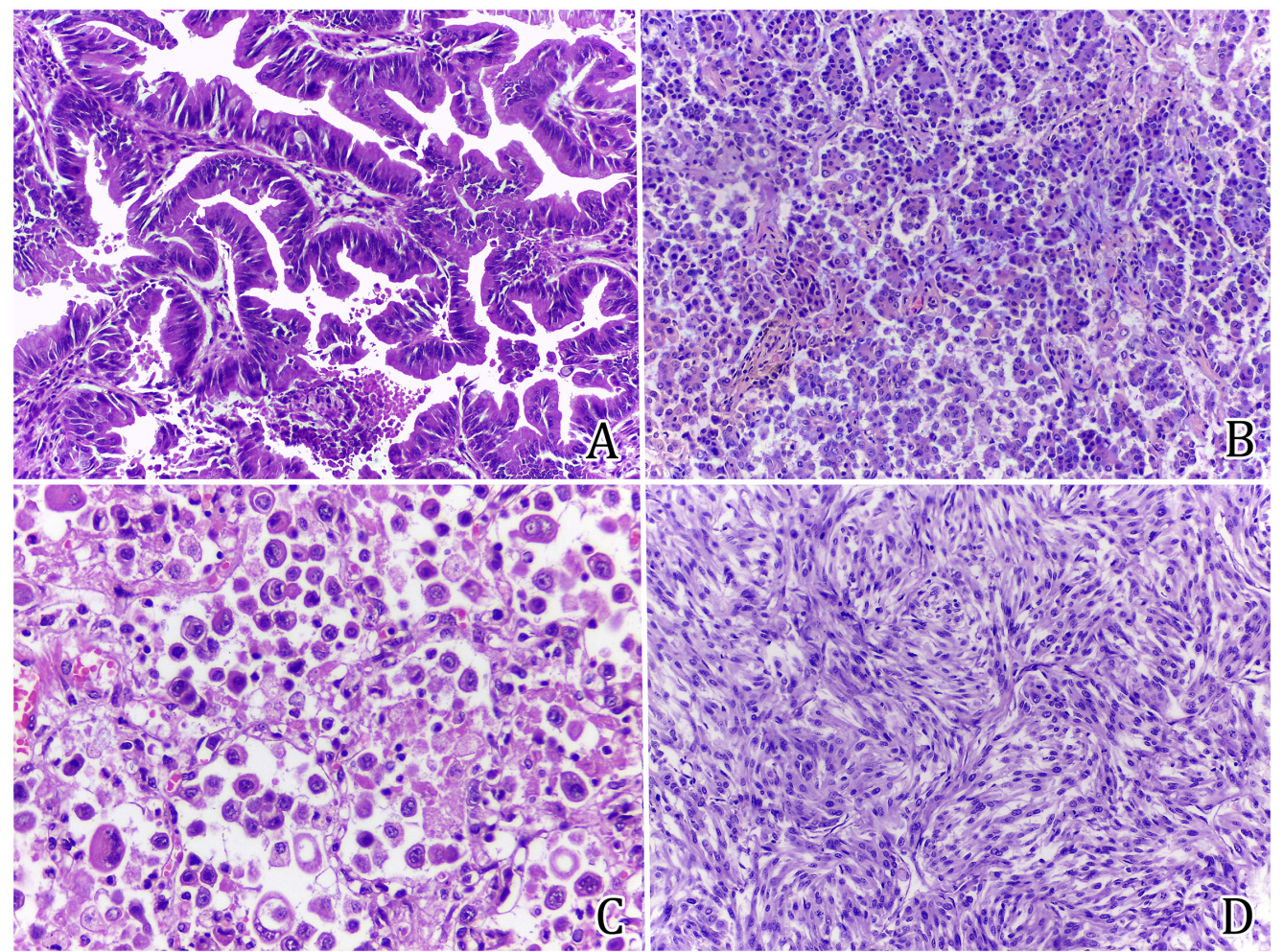

Fig.2. Aspectos histopatológicos dos principais neoplasmas pulmonares primários de cães diagnosticados no SPV/UFRGS. (A) Adenocarcinoma papilar, caracterizado por proliferação neoplásica que invadia e destruía o parênquima pulmonar, substituindo-o pelo padrão papilar. HE, obj.40x. (B) Carcinoma lepídico com células neoplásicas em espaços alveolares. HE, obj.40x. (C) Carcinoma de grandes células com substituição do parênquima pulmonar por células neoplásicas grandes. HE, obj.40x. (D) Tumor da bainha de nervo periférico maligno caracterizado por proliferação de células neoplásicas dispostas em feixes. HE, obj.40x.. 
observadas moderada necrose intratumoral $(2 / 5 ; 40 \%)$, invasão de vasos $(5 / 5 ; 100 \%)$, discreta hemorragia $(3 / 5$; $60 \%$ ) e fendas de colesterol (1/5; 20\%) (Fig.2C).

O caso de carcinoma adenoescamoso $(1 / 36)$ possuía células neoplásicas dispostas em ninhos, eram cuboidais a alongadas, ocasionalmente, dispostas em paliçada, com citoplasma eosinofílico, moderadamente delimitado, núcleo redondo a alongado, cromatina frouxa e nucléolo evidente. Havia moderado pleomorfismo e até oito figuras de mitose por campo de maior aumento (400x). Em meio ao tumor havia diferenciação escamosa multifocal e extensas áreas de necrose e hemorragia.

O carcinoma de células escamosas $(1 / 36)$ se caracterizou por células tumorais organizadas em ninhos sustentados por escasso estroma fibroso, com moderada diferenciação escamosa, e por vezes contendo pouca quantidade de material lamelar eosinofílico (pérolas de queratina). As células eram redondas, com citoplasma eosinofílico, bem delimitado, núcleo redondo a ovalado e nucléolo evidente. Havia discreto pleomorfismo e não foram observadas figuras de mitose. Menos frequentemente foi notada invasão de vasos.

O carcinoma combinado $(1 / 36)$ foi subdividido em adenocarcinoma acinar e carcinoma de pequenas células. Este último se caracterizava por formar pequenos lóbulos entremeados por discreto estroma fibrovascular. As células eram poligonais, com citoplasma escasso, núcleo redondo e nucléolo evidente. Havia discreto pleomorfismo e raras figuras de mitose. Havia necrose intratumoral discreta, hemorragia e invasão de vasos.

Os dois tumores de bainha de nervo periférico maligno $(2 / 36 ; 5,5 \%)$ foram definidos por possuírem células neoplásicas dispostas em feixes e sustentadas por discreto estroma fibroso $(2 / 2 ; 100 \%)$. As células eram fusiformes, com citoplasma eosinofílico, escasso e bem delimitado, núcleo alongado e nucléolo evidente. Havia moderado pleomorfismo $(2 / 2 ; 100 \%)$ e em média de 4 a 6 figuras de mitose por campo de maior aumento (400x) (Fig.2D). Em um dos casos havia moderada necrose intratumoral.

\section{Achados imuno-histoquímicos}

$\mathrm{Na} I H Q$, todos os tumores epiteliais $(34 / 34 ; 100 \%)$ marcaram para pancitoqueratina no citoplasma (Fig.3A). Houve marcação intranuclear de fator de transcrição de tireoide-1 (TTF-1) em 70,6\% dos casos (Fig.3B). Diferentes graduações foram observadas, onde houve discreta $(11 / 34 ; 32,3 \%)$, moderada $(7 / 34 ; 20,6 \%)$ e acentuada $(6 / 34 ; 17,6 \%)$ marcação, com a seguinte ordem decrescente de marcação: adenocarcinoma, carcinoma lepídico, carcinoma de células escamosas, carcinoma adenoescamoso, carcinoma combinado e carcinoma de grandes células. Em dez casos não houve marcação para TTF-1. Tumores epiteliais testados para vimentina obtiveram marcação positiva em oito casos (23,5\%) (Fig.3C), sendo que somente um dos tumores foi moderadamente marcado, e o restante obteve marcação discreta. Os dois tumores

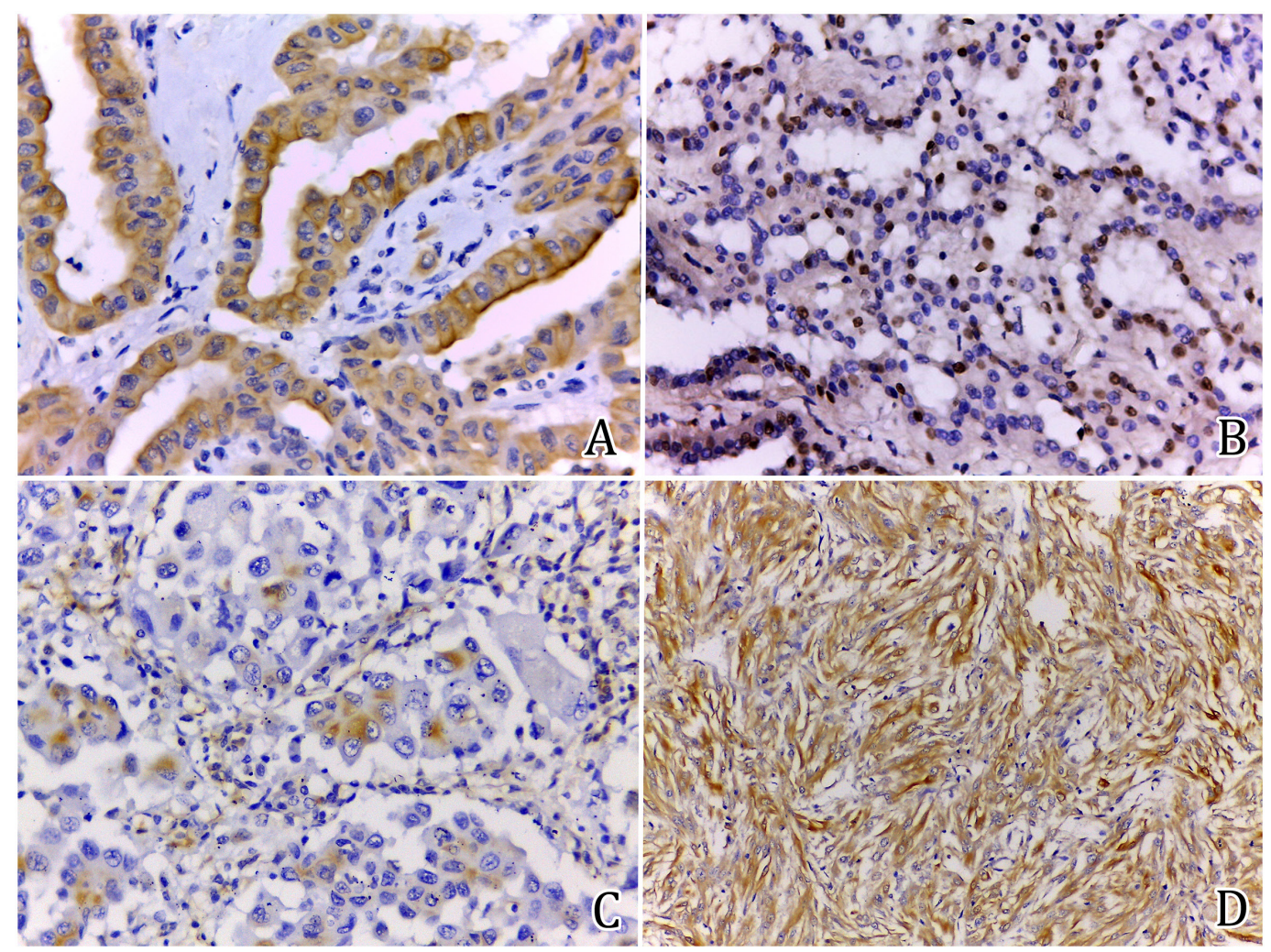

Fig.3. Achados imuno-histoquímicos dos principais neoplasmas pulmonares primários de cães diagnosticados no SPV/UFRGS. (A) Imunomarcação intracitoplasmática para pancitoqueratina em um caso de adenocarcinoma papilar. Obj.40x. (B) Imunomarcação nuclear de TTF-1 em um caso de adenocarcinoma papilar. Obj.40x. (C) Imunomarcação intracitoplasmática para vimentina em um caso de carcinoma lepídico. Obj.40x. (D) Imunomarcação intracitoplasmática para S-100 em um caso de tumor da bainha de nervo periférico maligno. Obj.40x. 
Quadro 3. Imunomarcação de neoplasmas pulmonares primários de origem epitelial para pancitoqueratina, TTF-1 e vimentina

\begin{tabular}{lccc}
\hline \multicolumn{1}{c}{ Tumor epitelial pulmonar canino } & Pancitoqueratina & TTF-1 & $1 / 18(5,5 \%)$ \\
\hline Adenocarcinoma & $18 / 18(100 \%)$ & $14 / 18(77,8 \%)$ & 0 \\
Adenocarcinoma papilar & $11 / 11(100 \%)$ & $9 / 11(81,8 \%)$ & 0 \\
Adenocarcinoma acinar & $2 / 2(100 \%)$ & $1 / 2(50 \%)$ & 0 \\
Adenocarcinoma sólido & $1 / 1(100 \%)$ & $0 / 1(0 \%)$ & $1 / 18(5,5 \%)$ \\
Adenocarcinoma misto & $4 / 4(100 \%)$ & $6 / 8(100 \%)$ & $4 / 8(50 \%)$ \\
Carcinoma lepídico & $8 / 8(100 \%)$ & $1 / 5(20 \%)$ & $3 / 5(60 \%)$ \\
Carcinoma de grandes células & $5 / 5(100 \%)$ & $1 / 1(100 \%)$ & 0 \\
Carcinoma de células escamosas & $1 / 1(100 \%)$ & $1 / 1(100 \%)$ & 0 \\
Carcinoma adenoescamoso & $1 / 1(100 \%)$ & $1 / 1(100 \%)$ & 0 \\
Carcinoma combinado & $1 / 1(100 \%)$ & &
\end{tabular}

TTF-1 = thyroid transcription factor-1.

mesenquimais do estudo foram positivos para vimentina e S-100 (Fig.3D) e negativos para actina de músculo liso e melan-A. Na técnica histoquímica de tricrômico de Masson, os tumores mesenquimais também foram negativos. Maiores informações referentes à imuno-histoquímica são descritas no Quadro 3.

\section{DISCUSSÃO}

O número de mortes por neoplasmas malignos em cães e gatos vem aumentando, o que tornou esta enfermidade uma causa de morte comum e frequente em animais de companhia (Rosseto et al. 2009). Este aumento, em suma, é decorrente do aumento da expectativa de vida dos animais, assim como da evolução dos métodos diagnósticos, aumento do número de amostras enviadas aos laboratórios de patologia veterinária e pelo reflexo da contaminação ambiental por inúmeros poluentes (Moulton et al. 1981, Mehlhaff \& Mooney 1985). Na América do Norte e Europa a frequência de NPP em cães varia de 0,1\% (Nielsen 1968) a 0,9\% (Stunzi et al. 1974), corroborando com a frequência de $0,5 \%$ deste estudo. Na medicina humana foi estimado que 29\% das mortes são relacionadas à ocorrência de tumores pulmonares, sendo estes atualmente a causa de mortalidade por câncer mais comum no mundo todo (Husain 2010).

Os neoplasmas mais diagnosticados neste estudo foram o adenocarcinoma e o carcinoma lepídico, com uma frequência de $50 \%$ e 22,2\%, respectivamente. Dados estes que corroboram com a literatura (Ogilvie et al. 1989, Hahn et al. 1996, Wilson 2017, Ramos-Vara et al. 2005, Polton et al. 2008). Diversos pesquisadores relatam que a frequência de adenocarcinomas variou de 74,8\% a 80\% (Miles 1988, Ogilvie et al. 1989), média superior ao encontrado neste trabalho, e com relação aos carcinomas lepídicos, a frequência variou de $15 \%$ a $25,7 \%$ (Moulton et al. 1981, Ogilvie et al. 1989, Hahn et al. 1996, Polton et al. 2008), dado semelhante a frequência encontrada. Assim como neste estudo, os outros tipos de NPP foram menos frequentes em cães, diferente de humanos, em que o carcinoma de células escamosas e o carcinoma anaplásico são os mais frequentes (Wilson 2017).

Ao contrário do encontrado, em que o número de machos foi maior que o de fêmeas, alguns autores relatam haver NPP igualmente em animais de ambos os sexos (Miles 1988, Ettinger \& Feldman 2004), já outros autores acreditam que fêmeas são mais acometidas que machos (Ogilvie et al. 1989, McNiel et al. 1997, Polton et al. 2008). A média de idade dos cães deste estudo foi de 11 anos, de acordo com a literatura em que a média varia entre nove e 12 anos de idade (Ogilvie et al. 1989, McNiel et al. 1997, Wilson 2017, Ettinger \& Feldman 2004). Neste estudo, os cães SRD foram os mais acometidos, provavelmente pelo fato de que há maior incidência na clínica de animais sem raça definida na região metropolitana de Porto Alegre.

No presente estudo, 11 casos (30,5\%) fizeram metástases; adenocarcinoma e o carcinoma de grandes células foram os mais metastáticos. Em estudos prévios, 23\% de NPP fazem metástases (Griffey et al. 1998) e os tumores com metástase mais frequentes foram os carcinomas de células escamosas e os carcinomas anaplásicos, e menos frequentemente os adenocarcinomas e carcinomas lepídicos (McNiel et al. 1997). Foram encontradas metástases de NPP em linfonodo mediastínico, traqueobrônquico e adrenal, e menos comumente em fígado, baço, rim, encéfalo, hipófise, tireoide e esôfago. Já foi relatado metástase de NPP em linfonodos mediastínicos, olhos, costelas, adrenais, cérebro, fígado, coração e glândula perianal (Ogilvie et al. 1989, McNiel et al. 1997, Wilson 2017, Silva et al. 2012). Em humanos há citações de metástases em linfonodos, fígado, adrenal, ossos, cérebro, pele e medula espinhal (Woods 4th \& Koretz 1990, Fernandez et al. 2002). Quanto à localização, a forma multifocal foi a mais frequente. $\mathrm{Na}$ forma focal, a maior ocorrência foi no lobo caudal direito semelhante ao descrito por Wilson ( 2017), que relatam que os neoplasmas pulmonares frequentemente afetam o lado direito mais do que o lado esquerdo, porém, Morris \& Dobson (2007) relatam que ambos os lados são igualmente afetados.

A imuno-histoquímica para TTF-1 é um método altamente específico e moderadamente sensível para identificação de NPP em caninos (Ramos-Vara et al. 2005). Em estudo realizado por Ramos-Vara et al. (2005), com 120 tumores epiteliais pulmonares primários caninos, 64,2\% dos tumores apresentaram marcação para o TTF-1. Desses, o carcinoma lepídico e o carcinoma broncogênico foram os mais reativos. Neste mesmo estudo houve uma variação quanto à imunoreatividade dependendo do tipo de tumor e foram detectados por ordem decrescente de frequência: carcinoma broncogênico, carcinoma lepídico, adenocarcinoma e carcinoma adenoescamoso. Os carcinomas de grandes células e o carcinoma de células escamosas, além 
dos neoplasmas mesenquimais, não tiveram imunomarcação para TTF-1. Porém, em contrapartida, em estudo realizado por Hiroshima et al. (2006), houve marcação de TTF-1 para carcinoma de grandes células, assim como adenocarcinoma e carcinoma de pequenas células e não houve imunomarcação para carcinoma de células escamosas. No presente estudo, a porcentagem de tumores imunomarcados foi discretamente superior ao apresentado por Ramos-Vara et al. (2005), perfazendo $70,6 \%$ dos casos, sendo que a imunomarcaçao seguiu a seguinte ordem decrescente: adenocarcinoma, carcinoma lepídico, carcinoma de células escamosas, carcinoma adenoescamoso, carcinoma combinado e carcinoma de grandes células.

A coexpressão da imuno-histoquímica de citoqueratina e vimentina são características de tumores como mesotelioma, melanoma amelanótico, carcinoma renal, carcinoma prostático canino, carcinoma hepatocelular canino e adenocarcinoma broncogênico felino (Martin de las Mulas et al. 1995, Shiga \& Shirota 2000, Grieco et al. 2003). Esta coexpressão em NPP é raramente reportada na literatura veterinária. Neste estudo a frequência de coexpressão foi de 23,5\%. Em estudo realizado por Burgess \& Kerr (2009), em que 21 cães tinham tumores epiteliais pulmonares, oito foram positivos na IHQ para vimentina e citoqueratina, perfazendo 38\%, média superior ao encontrado no presente trabalho. Na literatura humana a frequência variou de 38 a 50\% (Gatter et al. 1987, Ordonez 2003). De acordo com Burgess \& Kerr (2009), a teoria de transição de células epiteliais para mesenquimais é o mecanismo que explicaria a origem de mestástases. Para disseminar, as células neoplásicas epiteliais que são coesas, perderiam a estabilidade e se transformariam em células mesenquimais, capazes de se disseminar. Portanto, esta teoria explicaria a coexpressão de citoqueratina e vimentina.

Tumores da bainha de nervo periférico maligno de pulmão, é extremamente raro em animais (Dungworth et al. 1999) e em humanos (Togashi et al. 2003, Domen et al. 2010). Como diagnóstico diferencial de tumor da bainha de nervo periférico maligno podem ser incluídos fibrossarcoma, hemangiopericitoma e melanoma amelanótico (Boos et al. 2015). A imunomarcação para proteína S-100 é importante para diferenciar tumor da bainha de nervo periférico de fibrossarcoma, pois o último não exibe marcação para esta proteína (Gross et al. 2009). Neste estudo, além da marcação para S-100 também foi realizada a técnica histoquímica de tricrômico de Masson, na qual não houve identificação de colágeno, que também descarta o fibrossarcoma. A imunomarcação para actina de músculo liso pode indicar hemangiopericitoma, que neste caso foi negativa, e exclui este possível diagnóstico (Gaitero et al. 2008). 0 melanoma amelanótico tende a imunomarcar para melan-A e proteína relacionada à tirosinase-2 (Choi \& Kusewitt 2003) e neste estudo a IHQ para melan-A foi negativa, o que também descarta este diagnóstico diferencial.

\section{CONCLUSÕES}

Esse estudo, abordando epidemiologia, aspectos anatomopatológicos e imuno-histoquímicos em caninos com neoplasmas pulmonares primários (NPP) no período de janeiro de 2003 a junho de 2016, demonstrou que a média de idade de cães diagnosticados com NPP foi de 11 anos de idade. 0 número de machos afetados foi superior ao de fêmeas.

Adenocarcinoma pulmonar foi o NPP mais frequente, seguido do carcinoma lepídico. Dos principais tumores observados, os adenocarcinomas se caracterizaram por invadir e destruir o parênquima pulmonar, enquanto que nos carcinomas lepídicos haviam células neoplásicas em espaços alveolares.

Na imuno-histoquímica, todos os tumores epiteliais foram marcados para pancitoqueratina, 70,6\% foram positivos para TTF-1 e 23,5\% tiveram coexpressão para pancitoqueratina e vimentina. Tumores pulmonares mesenquimais foram raros em comparação com tumores epiteliais. 0 tumor da bainha de nervo periférico maligno encontrado é um tumor de origem pulmonar extremamente raro, tanto em animais quanto em humanos, e na IHQ apresenta marcação positiva para vimentina e S-100.

\section{REFERÊNCIAS}

Boos G.S., Bassuino D.M., Wurster F., Castro N.B., Watanabe T.T.N., Silva G.S., Sonne L. \& Driemeier D. 2015. Retrospective canine skin peripheral nerve sheath tumors data with emphasis on histologic, immunohistochemical and prognostic factors. Pesq. Vet. Bras. 35(12):965-974. http://dx.doi. org/10.1590/S0100-736X2015001200005.

Burgess H.J. \& Kerr M.E. 2009. Cytokeratin and vimentin co-expression in 21 canine primary pulmonary epithelial neoplasms. J. Vet. Diagn. Invest. 21(6):815-820. http://dx.doi.org/10.1177/104063870902100607. PMid:19901281.

Choi C. \& Kusewitt D.F. 2003. Comparison of tyrosinaserelated protein-2 S-100 and Melan-A immunoreactivity in canine amelanotic melanomas. Vet. Pathol. 40(6):713-718. http://dx.doi.org/10.1354/vp.40-6-713. PMid:14608029.

Domen H., Iwashiro N., Kimura N., Jinushi E., Komuro K., Ohara M. \& Ishizaka M. 2010. Intrapulmonary cellular schwannoma. Ann. Thorac. Surg. 90(4):1352-1355. http://dx.doi.org/10.1016/j.athoracsur.2010.04.023. PMid:20868846.

Dungworth D.L., Hauser B., Hahn F.F., Wilson D.W., Haenichen T. \& Harkema J.R. 1999. Histological Classification of Tumors of the Respiratory System of Domestic Animals. Armed Forces Institute of Pathology, Washington, DC, p.16-38.

Ettinger S. \& Feldman E.C. 2004. Textbook of Veterinary Internal Medicine. 5th ed. W.B. Saunders, Philadelphia, p.1120-1151.

Fernandez A., Jatene F.B. \& Zamboni M. 2002. Diagnóstico e estadiamento do câncer de pulmão. J. Bras. Pneumol. 28(4):219-228. http://dx.doi. org/10.1590/S0102-35862002000400006.

Gaitero L., Añor S., Fondevila D. \& Pumarola M.. 2008. Canine cutaneous spindle cell tumours with features of peripheral nerve sheath tumours: a histopathological and immunohistochemical study. J. Comp. Pathol. 139(1):16-23. http://dx.doi.org/10.1016/j.jcpa.2008.03.003. PMid:18514218

Gatter K.C., Dunnill M.S., Heryet A. \& Mason D.Y. 1987. Human lung tumours: does intermediate filament co-expression correlate with other morphological or immunocytochemical features? Histopathology 11(7):705-714. http:// dx.doi.org/10.1111/j.1365-2559.1987.tb02684.x. PMid:3040564.

Grieco V., Patton V., Romussi S. \& Finazzi M. 2003. Cytokeratin and vimentin expression in normal and neoplastic canine prostate. J. Comp. Pathol. 129(1):78-84. http://dx.doi.org/10.1016/S0021-9975(03)00006-9. PMid:12859911.

Griffey S.M., Kraegel S.M. \& Madewell B.R. 1998. Rapid detection of K-ras gene mutation in canine lung cancer using single-strand conformational polymorphism analysis. Carcinogenesis 19(6):959-963. http://dx.doi. org/10.1093/carcin/19.6.959. PMid:9667731.

Gross T.L., Ihrke P.J., Walder J.E. \& Affolter K.V. 2009. Doenças de Pele do Cão e do Gato: Diagnóstico Clínico e Histopatológico. 2 $2^{\text {a }}$ ed. Roca, São Paulo, p.770-780. 
Hahn F.F., Muggenburg B.A. \& Griffith W.C. 1996. Primary lung neoplasia in a beagle colony. Vet. Pathol. 33(6):633-638. http://dx.doi. org/10.1177/030098589603300601. PMid:8952021.

Hiroshima K., Iyoda A., Shida T., Shibuya K., Iizasa T., Kishi H., Tanizawa T., Fujisawa T. \& Nakatani Y. 2006. Distinction of pulmonary large cell neuroendocrine carcinoma from small cell lung carcinoma: a morphological, immunohistochemical, and molecular analysis. Modern Pathology 19(10):1358-1368. http://dx.doi.org/10.1038/modpathol.3800659. PMid:16862075.

Husain A.N. 2010. O pulmão, p.685-770. In: Kumar V., Abbas A.K., Fausto N. \& Aster J.C. (Eds), Patologia: Bases Patológicas das Doenças. 8aa ed. Elsevier, Rio de Janeiro.

Martín de las Mulas J., Espinosa de los Monteros A., Carrasco L., Van Niel M. \& Fernández A. 1995. Immunohistochemical distribution pattern of intermediate filament proteins in 50 feline neoplasms. Vet. Pathol. 32(6):692701. http://dx.doi.org/10.1177/030098589503200611. PMid:8592805.

McNiel E.A., Ogilvie G.K., Powers B.E., Hutchison J.M., Salman M.D. \& Withrow S.J. 1997. Evaluation of prognostic factors for dogs with primary lung tumors: 67 cases (1985-1992). J. Am. Vet. Med. Assoc. 211(11):1422 1427. PMid:9394893.

Mehlhaff C.J. \& Mooney S. 1985. Primary pulmonary neoplasia in the dog and cat. Vet. Clin. N. Am., Small Anim. Pract. 15(5):1061-1067. http://dx.doi. org/10.1016/S0195-5616(85)50110-2. PMid:3878026.

Miles K.G. 1988. A review of primary lung tumors in the dog and cat. Vet. Radiol. 29(3):122-128. http://dx.doi.org/10.1111/j.1740-8261.1988.tb01761.x

Morris J. \& Dobson J. 2007. Oncologia em Pequenos Animais. Roca, São Paulo, p.145-154.

Moulton J.E., Von Tscharne C. \& Schneider R. 1981. Classification of lung carcinomas in the dog and cat. Vet. Pathol. 18(4):513-528. http://dx.doi. org/10.1177/030098588101800409. PMid:6266118.

Nielsen W. 1968. Comparative pathology of pulmonary disease, p.226-244 In: Liebow A.A. \& Smith D.E. (Eds), The Lung. Williams and Wilkins Co. Baltimore, MD.

Ogilvie G.K., Haschek W.M., Withrow S.J., Richardson R.C., Harvey H.J., Henderson R.A., Fowler J.D., Norris A.M., Tomlinson J., McCaw D., Klausner J.S., Reschke R.W. \& McKiernan B.C. 1989. Classification of primary lung tumors in dogs: 210 cases (1975-1985). J. Am. Vet. Med. Assoc. 195(1):106108. PMid:2547742.

Ordóñez N.G. 2003. The immunohistochemical diagnosis of mesothelioma: a comparative study of epitheliod mesothelioma and lung adenocarcinoma.
Am. J. Surg. Pathol. 27(8):1031-1051. http://dx.doi.org/10.1097/00000478200308000-00001. PMid:12883236.

Polton G.A., Brearley M.J., Powell S.M. \& Burton C.A. 2008. Impact of primary tumour stage on survival in dogs with solitary lung tumours. J. Small Anim. Pract. 49(2):66-71. http://dx.doi.org/10.1111/j.1748-5827.2007.00403.x. PMid:17725584.

Priebe A.P.S., Riet-Correa G., Paredes L.J.A., Costa M.S.F., Silva C.D.C. \& Almeida M.B. 2011. Ocorrência de neoplasias em cães e gatos da mesorregião metropolitana de Belém, PA entre 2005 e 2010. Arq. Bras. Med. Vet. Zootec. 63(6):1583-1586. http://dx.doi.org/10.1590/S0102-09352011000600042.

Ramos-Vara J.A., Miller M.A. \& Johnson G.C. 2005. Usefulness of thyroid transcription factor-1 immunohistochemical staining in the differential diagnosis of primary pulmonary tumors of dogs. Vet. Pathol. 42(3):315320. http://dx.doi.org/10.1354/vp.42-3-315. PMid:15872377.

Reif J.S., Dunn K., Ogilvie G.K. \& Harris C.K. 1992. Passive smoking and canine lung cancer risk. Am. J. Epidemiol. 135(3):234-239. http://dx.doi. org/10.1093/oxfordjournals.aje.a116276. PMid:1546698.

Rosseto V.J.V., Moreno K., Grotti C.B., Reis A.C.F. \& Bracarense A.P.F.R.L. 2009. Frequência de neoplasmas em cães diagnosticados por exame citológico: estudo retrospectivo em um hospital-escola. Semina, Ciênc. Agrárias $30(1): 189-200$.

Shiga A. \& Shirota K. 2000. Vimentin/cytokeratin coexpression foci in a well-differentiated canine hepatocellular carcinoma. J. Vet. Med. Sci. 62(2):199-202. http://dx.doi.org/10.1292/jvms.62.199. PMid:10720192.

Silva E.O., Green K.T., Wasques D.G., Reis A.C.F. \& Bracarense A.P.F.R.L. 2012. Tumor primário pulmonar metastático em três cães. Semina, Ciênc. Agrárias 33(2):3271-3278.

Stunzi H., Head K.W. \& Nielsen S.W. 1974. Tumors of lung. Bulletin WHO 50(1/2):9-19. PMid:4371738.

Togashi K., Hirahara H., Sugawara M. \& Oguma F. 2003. Primary malignant schwannoma of the lung. Jpn. J. Thorac. Cardiovasc. Surg. 51(12):692-695. http://dx.doi.org/10.1007/s11748-003-0014-7. PMid:14717429.

Wilson D.W. 2017. Tumors of the respiratory tract, p.467-498. In: Meuten D.J. (Ed), Tumors in Domestic Animals. 5th ed. John Wiley and Sons, New Jersey.

Woods 4th J.M. \& Koretz M.J. 1990. Emergency abdominal surgery for complications of metastatic lung carcinoma. Arch. Surg. 125(5):583-585. http://dx.doi.org/10.1001/archsurg.1990.01410170029005. PMid:2158777. 